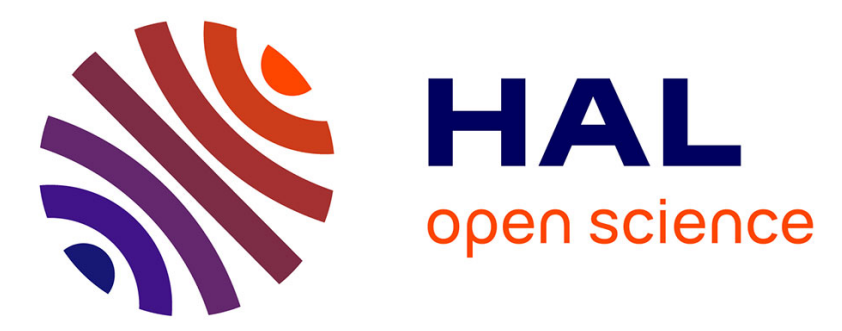

\title{
Superconducting magnetometers
}

\author{
J.E. Mercereau
}

\section{- To cite this version:}

J.E. Mercereau. Superconducting magnetometers. Revue de Physique Appliquée, 1970, 5 (1), pp.13-

20. 10.1051/rphysap:019700050101300 . jpa-00243347

\section{HAL Id: jpa-00243347 https://hal.science/jpa-00243347}

Submitted on 1 Jan 1970

HAL is a multi-disciplinary open access archive for the deposit and dissemination of scientific research documents, whether they are published or not. The documents may come from teaching and research institutions in France or abroad, or from public or private research centers.
L'archive ouverte pluridisciplinaire HAL, est destinée au dépôt et à la diffusion de documents scientifiques de niveau recherche, publiés ou non, émanant des établissements d'enseignement et de recherche français ou étrangers, des laboratoires publics ou privés. 


\title{
SUPERGONDUGTING MAGNETOMETERS
}

\author{
By J. E. MERGEREAU, \\ California Institute of Technology, Alfred. P. Sloan Laboratory of Mathematics and Physics, \\ Pasadena, California (U.S.A.).
}

\begin{abstract}
Under the proper circumstances a current carrying superconductor can generate voltage. This voltage is produced by supercurrent oscillations at frequency $\omega=\frac{2 e}{\hbar} V-$ a phenomena similar to the Josephson effect in superconducting tunnel junctions. Various structures have been fabricated in which this quantum relation dominates the electrical behavior. These thin film structures have been developed into reliable sensor elements which have been incorporated into various electronic instruments - particularly magnetometers. These instruments include a differential magnetometer with a one second response time and sensitivity greater than $10^{-10}$ gauss and a digital magnetometer counting in increments of $1 / 4 h / 2 e$ at a rate up to $10^{4}$ per second. The fundamental principles of these instruments will be discussed as well as their present operational limitations.
\end{abstract}

Zero electrical resistance has been the principal hallmark of the phenomena of superconductivity. It was, in fact, this effect which alerted Kamerlingh Onnes that he has uncovered a new physical phenomenon. This electrical aspect of superconductivity, the ability to carry finite current at zero voltage has received major attention from experimentalists for some time. This is primarily because there is at least an adequate phenomenological description of this aspect of superconductivity and also, in a practical sense, because of the vast economic implications of low cost power transmission and very high field superconducting magnets.

However, there is another facet of superconductivity which in the past seems to have received somewhat less attention. Despite the apparent contradiction in terms, this kind of superconductivity can probably best be characterized as the resistive superconducting state - that is to say, superconductivity at finite voltages. In contrast to zero voltage superconductivity there seems to be little theory to guide the experimenter into this intrinsically time dependent problem. The purpose of this paper is to indicate our use of superconductivity at finite voltages for instrumentation purposes and, in the absence of a more complete theory, to attempt to describe our results in physical terms.

In order to sustain a voltage supporting superconducting state it is usually necessary that the super-electron density be inhomogeneous. Kim [1] and Gieaver [2] have studied this state in situations where the inhomogeneities are vortex lines produced by an external field. I want to concentrate here on the "field free" case where the inhomogeneity is determined by the material itself. The extreme of this inhomogeneity is the Josephson [3] tunnel junction, with an insulating barrier. However this structure has already had extensive treatment and since it is relatively delicate is not uniquely suitable for instrumentation. In what is to follow I would like to specifically exclude this particular inhomogeneity and consider only electron density variations resulting from current through the Dayem [4] bridge or a nonhomogeneous metal [5].
Voltage-supporting superconductivity. - In figure 1, curve A shows a typical current-voltage characteristic across such a superconducting structure driven by a current source. For currents less than some temperature-dependent critical current, $I_{\mathrm{c} 1}$, current can flow

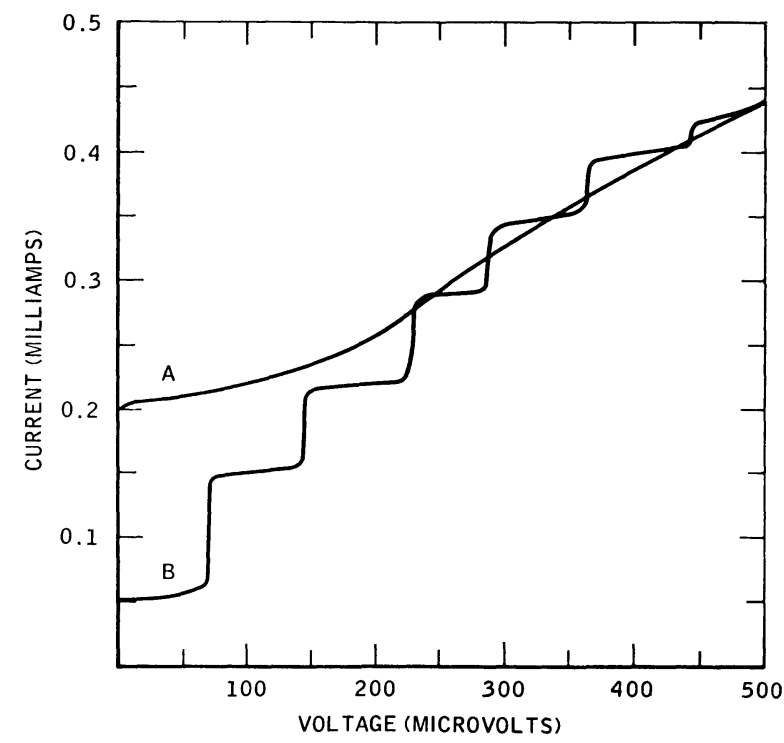

FIG. 1. - Voltage developed across a Dayem bridge driven from a current source. Curve A shows a critical current, $I_{\mathrm{c} 1}$, of about .2 milliamperes. Curve B is the same structure irradiated with microwaves at about $35 \mathrm{GHz}$.

without developing any voltage; while above this critical current, voltage $(V)$ exists. Voltage induced above, $I_{\mathrm{c} 1}$, indicates that power is being delivered to the superconductor from the current source. However, as I shall indicate, because of the peculiar process of voltage generation in a superconductor only part of this power is transformed into heat while the remainder can escape as high frequency radiation, principally at frequencies $\nu=N(2 e / h) V=N \varphi_{0}{ }^{-1} V$. 
These structures are deliberately fabricated in such a way that there is a definite, localized region of high current density. Within this region the electron drift velocity is considerably higher than in the surrounding superconductor. This usually means that the dimensions are small enough, relative to the penetration depth, that in the high density region the current can be assumed to be uniform. Consequently, in what follows we will use the concept of current and current density interchangeably. Current less than the critical value can, by definition, be accommodated without developing a voltage. However if the current supplied to the superconductor is greater than critical some other current transfer mechanism is required. What I shall do is to assume a two-fluid description for superconductivity and construct a model which gives rise to a possible mechanism.

On a two-fluid model we presume that for currents greater than critical, part of the current flows as supercurrent and part as normal current. The normal current requires voltage which is related to the normal current by the resistance, $V=I_{n} R$. Because of the geometry, $R$ is predominantly the resistance of the high current density region. If, above the critical current level, the superfluid component of the total current always remains at its maximum value $I_{c}$, the current-voltage characteristic would be as shown in figure 2, curve A; where we presume the voltage is developed only by the normal component of the current, $V=\left(I-I_{\mathrm{c}}\right) R$.

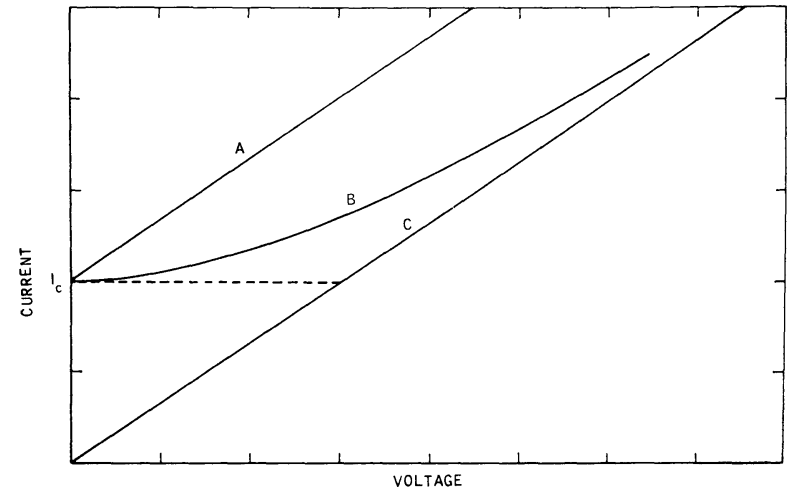

FIG. 2. - Idealized current-voltage characteristic for a Dayem bridge.

However, in the superconductor the relationship [6] between current density $j$ and electron drift velocity, $v$, is $j \propto v\left(1-v^{2} / v_{\mathrm{c}}^{2}\right)$. As a consequence, the maximum current density $j_{\mathrm{c}}$ does not occur at the maximum electron velocity, $v_{\mathrm{c}}$. At low velocities, as long as current increases with increasing electron velocity, a flux change will induce current to offset the change and the resultant Faraday induction will help to keep the magnetic field from collapsing and penetrating into the superconductor. On the other hand at high velocities $v>v_{\mathrm{c}} / \sqrt{3}$ current decreases with increasing electron velocity and an inverse Faraday induction [7] will occur, spontaneously driving the supercurrent and superelectron density to zero in a second order phase transition as the superelectron velocity reaches $v_{\mathrm{c}}$.

Therefore the previously described situation of the simultaneous flow of super and normal currents ( fig. 2, curve A) must not be a stable situation. The electric field, $E$, required to drive the normal current must also accelerate the superelectrons. On a free electron model, $\dot{v}=\frac{e}{m} E$. Thus the supercurrent cannot remain at $I_{c}$ in the presence of this electric field. The field will accelerate the superelectrons and drive the supercurrent to zero at a second order phase transition. In the process the voltage will have to increase in order to sustain the same total current.

If this sequence of events occurs, the current-voltage characteristic of the superconductor would be as shown on figure 2, curve C. As the current is slowly increased from zero it eventually exceeds $I_{\mathrm{c}}$ and a spontaneous transition will occur, driving the supercurrent component to zero, leaving the voltage to become $V=I R$. This voltage must be due to charge at the interfaces of the region of high current density. Since the region of high current density is bounded by equipotential surfaces (superconductor) these voltages must arise from an electrostatic potential across the bridge, creating a "linear capacitor".

However this situation is not stable either. If the system can maintain thermal equilibrium it will now recondense at zero velocity, since the electric field itself is not sufficient to quench superconductivity. The acceleration process will then repeat, part of the current becoming supercurrent, "discharging" the electric field until the electron drift velocity again reaches $v_{c}$, etc. If we assume that the inverse Faraday induction process occurs very rapidly we can characterize the period $(\tau)$ at which the process repeats as the time for the velocity to reach critical.

On a free electron model $E=\frac{m}{e} \dot{v}$, and thus :

$$
\tau \sim \frac{m v_{c}}{\langle E\rangle e}
$$

However, the definition of coherence length $\xi$ is such that :

$$
m v_{\mathrm{c}} \sim \frac{h}{\bar{\xi}}
$$

The coherence length by definition is the shortest distance over which the superelectron density can change and thus the smallest distance over which the field can exist. Thus if the geometry of the situation is such that the electric field exists only across a dimension equivalent to the coherence length the product $\langle E\rangle \xi$ must equal the voltage $V$, or $\tau \sim \hbar / 2 e V$. Actually this approximation must be exact since it is equivalent to the Josephson voltage-frequency relationship which must hold for superconductors in general. In this model $\tau^{-1}$ is the oscillation frequency of the supercurrent. Since the superconductor is driven from a current source, total current must be kept constant. Therefore the normal current component may also oscillate. And to the extent that $I_{\mathrm{s}}$ and $I_{\mathrm{n}}$ are not exactly of opposite time phase, there may also be displacement currents induced which represent possible radiative processes. As a result of these current "relaxation oscillations", voltage is generated due to the growth and decay of charge across the region of highest current density. Thus, this voltage also oscillates at frequency $\omega=\tau^{-1}$, but has an average value equal to $V=\hbar \omega / 2 e$. 
This average voltage thus depends on the time average of the supercurrent - ranging from curve A (fig. 2) when $I_{\mathrm{s}}=I_{\mathrm{c}}$ to curve $\mathrm{C}$ when $I_{\mathrm{s}}=0$. Experimentally a superconductor of this structure often shows a current-voltage characteristic such as that illustrated in figure 2, curve B. On the basis of this model the interpretation of curve $\mathrm{B}$ is that above $I_{\mathrm{c}}$ voltage appears, both to represent a decrease in $I_{\mathrm{s}}$ and also the appearance of radiated power. As the frequency of the supercurrent oscillation is increased the time average of the supercurrent component of the total current eventually decreases, probably because of some intrinsic relaxation time for supercurrent decay and recondensation. When finally the period of the relaxation oscillation is shorter than the intrinsic relaxation time, the supercurrent remains zero and we are back to curve $\mathrm{C}$.

Curve $\mathrm{B}$ in figure 1 gives some additional experimental justification for this model. In this circumstance the superconductor is being radiated by microwaves at frequency $\sim 35 \mathrm{GHz}$. The zero-voltage current is depressed since the threshold current level must now accommodate not only the bias current but also the high frequency current induced by the radiation. Above the threshold level any coupling whatever between the supercurrents and the microwaves would tend to make the supercurrent move at the microwave frequency. Thus the superconductor would tend to have certain prefered oscillation frequencies or voltages. These data show oscillation at the driving frequency and also, because of the non-linearity in this system, oscillation at frequencies up to the 5 th harmonic. The $n$th harmonic corresponds to $n$ relaxation oscillations per cycle of microwave frequency. Data such as this have been observed up to voltages of a few millivolts which corresponds to oscillation frequencies on the order $10^{12}$ cycles per second.

Structure such as this has also been observed in $I-V$ characteristics for tiny contacts between normal and superconducting metals [8], which thus cannot be interpreted in terms of the relative behavior of two superconductors, as in the usual Josephson effect. However this concept of supercurrent oscillation near the boundry can be used to explain these effects at a normal-super interface.

In summary then : in structures such as these, above some lower critical current $I_{\mathrm{c} 1}$, the supercurrent begins to undergo relaxation oscillations. The rate of supercurrent oscillation is determined by the average voltage and the average voltage in turn is determined by the relative time spent, during each cycle, in the superconducting or normal states. As the current is increased, the voltage or frequency increases, decreasing the amount of time spent in the superconducting state and the average voltage gradually increases with increasing current. This process appears to persist to frequencies at least as high as the energy gap in superconductor with no apparent degradation in the quantum relationship $\hbar \omega=2 \mathrm{eV}$. Thus the two-fluid model seems entirely adequate; the normal and supercurrents seem to enter only in an additive way with no interference effects.

There must exist an upper critical current $I_{\mathrm{c} 2}$ at which these dynamic superconducting processes cease. This may occur because of heating by the normal current or, more fundamentally, because of some frequency limitation such as the energy gap or other relaxation process. However in what follows we will concentrate only on the low frequency behavior and discuss devices incorporating this kind of superconductivity into a superconducting circuit.

Superconducting circuits. - It had been noticed sometime ago that under the right conditions a thin film superconducting ring is effectively transparent to magnetic flux [9]. If such a superconducting ring is placed in an axial field it will shield flux changes from its interior, but only up to some critical current $I_{\mathrm{c} 1}$, in the ring. For flux changes larger than this, current in the ring remains essentially constant while flux passes thru. Under these conditions the ring is in the previously described voltage-supporting superconducting state. In order to control this voltage, which is the basis for the instrumentation I will discuss, the ring itself is usually made non-homogeneous by means of a Dayem [4] bridge or a microscopic material inhomogeneity [5]. These configurations localize the flux flow region and allow us to experimentally control the flux motion.

Driving these rings inductively is equivalent to providing a voltage source rather than a current source, as in the previous discussion. When the induced current reaches critical in the bridge, it must nevertheless spontaneously decay, as before, and in the process create displacement current, $\dot{E}$, in the bridge. After the spontaneous decay the bridge can again condense but now in a finite electric field produced by the displacement current - this field then "discharges" and accelerates the current again to nearly the critical value. By arguments similar to those used before this process can be related to flux loss by the ring.

$$
\begin{gathered}
\text { Since } \dot{v}=\frac{e}{m} E=\frac{e}{m} \dot{A} \text { in the proper gauge, then : } \\
\delta v=\frac{e}{m} \delta A .
\end{gathered}
$$

Thus the spontaneous decay of current, which changes the velocity by roughly $v_{\mathrm{c}} / 2$ must be accomplished by a change in potential $\delta A \sim \frac{m v_{\mathrm{c}}}{2 e}$. Again substituting for $m v_{\mathrm{c}} \sim \frac{h}{\xi}$, we get $\xi \delta A \sim h / 2 e$. And if the superconductor actually manages to limit the current decay to the smallest possible distance $\xi$, the flux change, $\delta \varphi=\xi \delta A$ produced in the ring will be one quantum, $\varphi_{0}=h / 2 e$. This model thus envisages a situation in which individual flux quanta escape from the ring in a process which interchanges real and displacement currents. The final current, $I_{\mathrm{f}}$, in the ring after the transition must be sufficient to sustain the original flux, minus $\varphi_{0}$. Thus $I_{\mathrm{f}}=I_{\mathrm{c}}-\varphi_{0} / L$, where $L$ is the inductance of the ring.

The amplitude of the voltage pulse, $\delta V$, accompanying this transition depends on the relaxation time $\tau_{1}$ for the spontaneous decay, $\delta V=\varphi_{0} \tau_{1}^{-1}$. As an estimate for this time we draw upon Kim's results for current driven flux motion in superconductors [1]. He finds that flux moves perpendicular to the current at a rate $v_{1}=j \varphi_{0} / \eta$. If we assume that this viscosity [10] ( $\eta$ limited velocity can be related to a maximum rate of change for the order parameter then :

$$
\tau_{1} \sim \xi / v_{1} \text {. }
$$


At the critical current this reduces to :

$$
\tau_{1} \sim\left(\mu_{0} \sigma \lambda^{2}\right)=\hbar / \Delta
$$

where $\sigma$ and $\lambda$ are respectively the normal state conductivity and the superconducting penetration depth and $\Delta$ is the energy gap. For most of our circuits $\tau_{1}<10^{-11} \mathrm{~s}$.

This type of operation will occur only for bridge widths small enough that a flux vortex cannot be formed within the bridge - i.e. width less than a coherence distance. For larger bridges, current density is not uniform and is free to adjust internally and permit the formation of flux vortices within the bridge. If these flux quanta are subsequently removed by the current, via the Lorentz force, a quasi-continuous flux transfer can still occur giving rise to voltage by induction [4]. Operation of the wider bridges has been observed but in general is much more erratic that for small bridges, $w<\xi(T)$.

The magnetic flux enclosed by one of these superconducting rings is shown in figure $3 \mathrm{~A}$. This figure shows the magnetic flux enclosed by a superconducting ring as a function of the applied flux. This is the flux in the ring, presuming that the applied flux has started from zero and has been slowly increased. Up to some critical level, determined by the critical current of the bridge, the flux enclosed by the ring is held constant. Once this critical level is exceeded, flux begins to enter the ring as quanta. When a quantum enters a ring the flux shielded by the ring must also decrease by a quantum and the persistent current around the ring

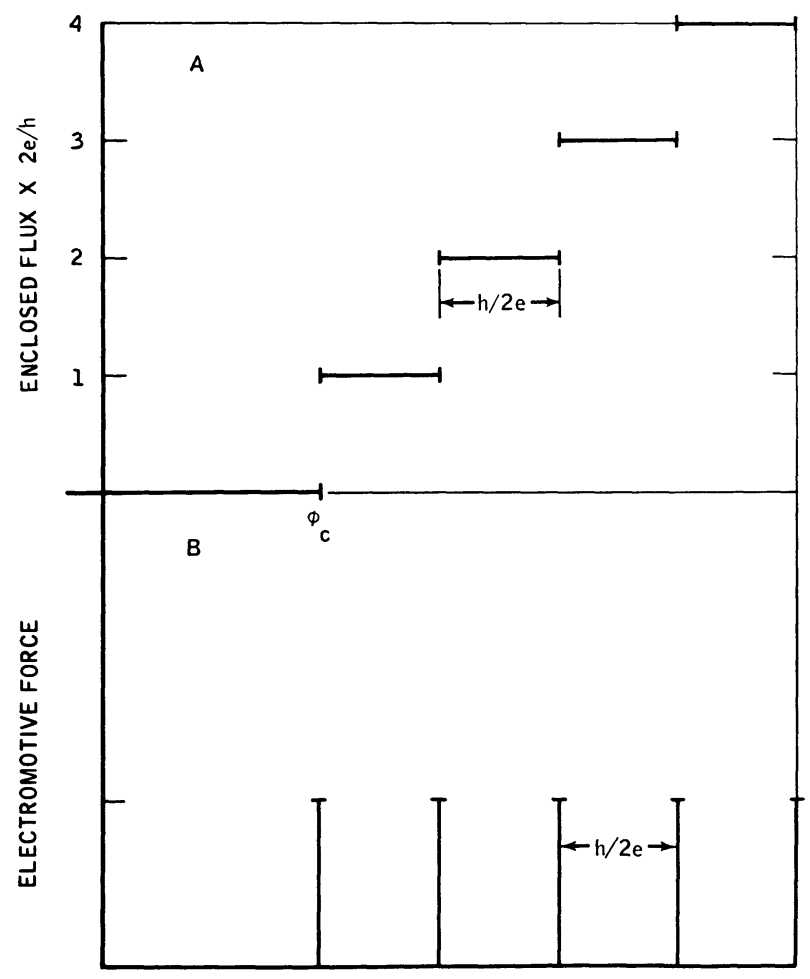

APPLIED FLUX

FIG. 3.

A) Magnetic flux enclosed by a thin film superconducting ring as a function of applied flux. Above $\varphi_{0}$ flux enters as quanta.

B) Emf generated by flux transfer into the ring as a function of applied flux. Pulse amplitude is determined by flux transit time. decreases by $\varphi_{0} L^{-1}$, where $L$ is the inductance of the ring. Thus the external flux must again be increased by an amount $\varphi_{0}$ to once more reach the critical condition at which the process repeats. Since the relaxation time is much shorter than any other characteristic time of our circuit, the emf produced by this flux change is shown in figure $3 \mathrm{~B}$. The time average of this emf is the voltage to which I referred in the previous discussion of the voltage supporting superconducting state. In the previous discussion the total current was held fixed and above some critical level the supercurrent sustained relaxation oscillations at a rate determined by the voltage. In the case of the inductively driven superconducting ring however the current is not fixed and is free to adjust to flux changes within the ring. The flux change in this case is not continuous since it is triggered only when the current reaches its critical value. As a consequence, this inductively driven ring acts as a "flux valve" producing an emf composed of a series of pulses of amplitude $\varphi_{0} \tau_{1}^{-1}$ spaced by increments, $\varphi_{0}$, in external flux.

In the experiments which I will describe, this emf is examined by observing the response of a tuned circuit driven by the superconducting ring. To illustrate this response we can Fourier analyze the pulse train using flux as the expansion variable and for the time being ignore the starting transients. To account for a possible finite relaxation time in the flux change we assume the emf to be a series of square pulses. However for convenience we convert this presumed shape dependence to time intervals where $\tau_{1}$ is the flux transit time and $T$ is the time between pulses. Then :

$$
\mathrm{emf} \sim \frac{\varphi_{0}}{\tau_{1}} \sum_{n} \frac{(-1)^{n}}{n} \sin \left(2 n \pi \frac{\tau_{1}}{T}\right) \cos \left(2 n \pi \frac{\varphi}{\varphi_{0}}\right) .
$$

In this approximation the superconducting ring generates an emf whose amplitude is fixed by the relaxation time but whose frequency depends periodically on the applied flux. In the limit $\tau_{1} \ll T$ the emf becomes independent of $\tau_{1}$ and equals :

$$
\operatorname{emf} \sim \frac{\varphi_{0}}{T} \sum_{n} \cos \left(2 n \pi \frac{\varphi}{\varphi_{0}}\right)
$$

depending only on the time between pulses. If the applied flux oscillates in time, $\varphi=\varphi_{\mathrm{rf}} \sin \omega t, T$ is no longer a constant but can be approximated by :

$$
T \sim \frac{1}{\omega} \varphi_{0} / \varphi_{\mathrm{rr}} .
$$

The only other change in the analysis is to introduce a phase factor to take account of possible variations in response to positive or negative fluxes. This variation corresponds to the possibility of persistent current from some steady ambient field. The resultant emf from a superconducting ring, driven into flux flow by an oscillating field, of amplitude $\varphi_{\mathrm{rf}}$ and frequency $\omega$, in a steady field $\varphi_{\mathrm{dc}}$ can be characterized by [11] :

$$
\mathrm{emf} \sim \varphi_{\mathrm{rf}} \omega J_{1}\left(2 \pi \frac{\varphi_{\mathrm{rf}}}{\varphi_{0}}\right) \cos \left(2 \pi \frac{\varphi_{\mathrm{dc}}}{\varphi_{0}}\right) \sin \omega t
$$

where we display only a component at the fundamental frequency. Thus the amplitude of this voltage depends periodically on the amplitude of both the steady and alternating components of magnetic flux. This voltage has the same functional dependence as though the 
ring were actually interrupted by a Josephson tunnel barrier. However it is important to realize that the only physical requirement here is flux quantization - not the Josephson tunneling phenomena. These structures are not "weakly coupled" in the sense of the Josephson tunnel junction but are dynamically uncoupled by the critical current.

Experimental results by Nisenoff on this induced voltage are shown in figure 4 . These data show the
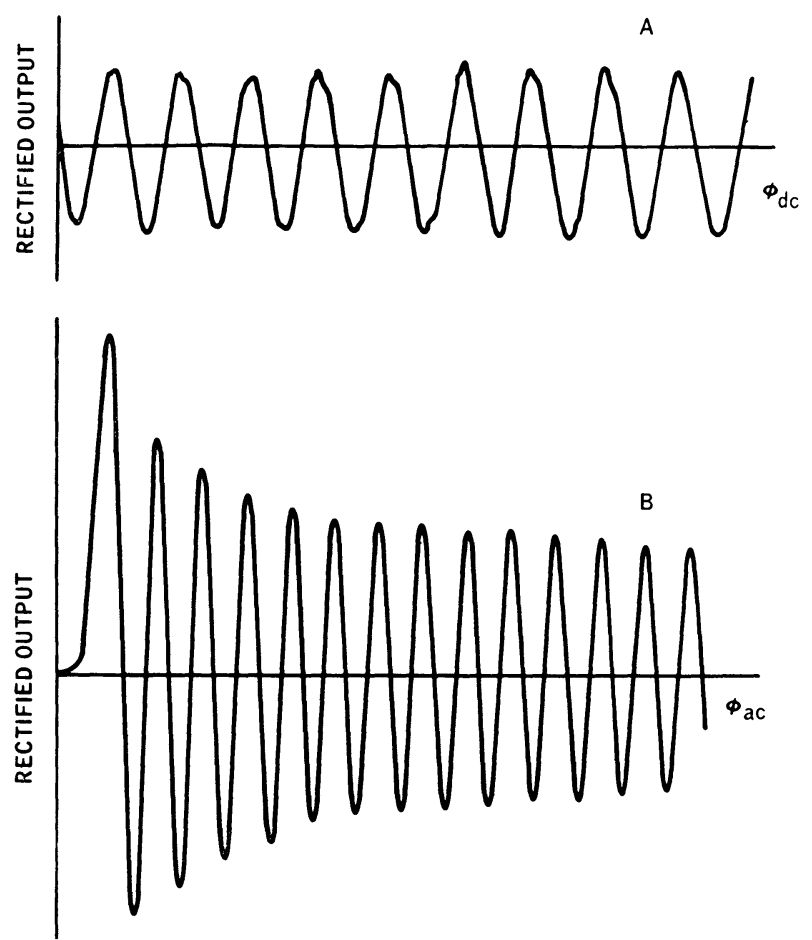

FIG. 4.

A) Amplitude of the superconducting emf as a function of steady flux, $\varphi_{\mathrm{de}}$. Pump amplitude, $\varphi_{\mathrm{rf}}$, is held constant.

B) Amplitude of the superconducting emf as a function of pump amplitude, $\varphi_{\mathrm{rf}}$. Steady field, $\varphi_{\mathrm{de}}$, is held constant. $\varphi_{\mathrm{ac}}$ and $\varphi_{\mathrm{de}}$ are on different scales - each is periodic in $h / 2 e$.

amplitude of an $\mathrm{rf}$ signal induced in a tank circuit inductively coupled to a superconducting thin film ring. Usually, because of interactions with the resonant tank circuit, feedback effects also connect the superconducting emf with $\varphi_{\mathrm{rf}}$ and act to limit the signal amplitude. If this feedback is phased properly the system can be made to oscillate. In these data the experimental variable is the applied magnetic flux, however the scale of the absciss differs for curves A and $\mathrm{B}$. Figure $4 \mathrm{~A}$ illustrates the variation of the signal amplitude at constant $\varphi_{\text {rf }}$ ("pump amplitude") corresponding to changes in the steady flux $\varphi_{\mathrm{dc}}$; where the elementary derivation also leads us to expect a sinusoidal variation. And, figure $4 \mathrm{~B}$ shows the signal amplitude as a function of pump amplitude when $\varphi_{\mathrm{dc}}$ is held fixed. In this case as long as $\tau_{1} \ll T$ the elementary derivation would lead us to expect a Bessel function type of oscillation. As the drive amplitude is increased the time between pulses decreases as $T \sim \varphi_{0}\left(\omega \varphi_{\mathrm{rf}}\right)^{-1}$. Ultimately the signal cuts off when there is no time between pulses to extract the quantum from the circuit or when $\omega t \sim \varphi_{0} / \varphi_{\mathrm{rf}}$.
These data were taken at a frequency of $30 \mathrm{MHz}$ on a thin film superconducting tin ring $300 \mathrm{~A}$ thick. This ring was formed by evaporation on a quartz rod $1 \mathrm{~mm}$ in diameter, the resulting ring being about a millimeter long. Into this ring was photo-etched a Dayem bridge about a micron wide. Data similar to this have been obtained from a number of superconducting configurations. The materials we have examined so far have been tin, lead, aluminum, niobium and various alloys of tin-lead and indium-tin. The width and thickness of the Dayem bridge largely determines the temperature of operation of the device. For a $300 \mathrm{~A}$ tin film and a bridge width of about a micron the device will operate from the transition temperature $(\sim 3.8 \mathrm{oK})$ down to at least $1.2 \mathrm{oK}$. Over this temperature range the critical current and thus the bias level increases with decreasing temperature. However at the lowest temperatures the device still operates even though the necessary bias level may be several thousand times greater than the signal. As the bridge width increases the temperature range of operation decreases, probably because of the requirement that $w<\xi(T)$. For a $300 \mathrm{~A}$ tin film the operating range is reduced to about 15 millidegrees for a $200 \mathrm{mi}$ cron bridge.

This degree of experimental confirmation of the concepts of the operation of these superconducting devices is about all we can expect due to the highly simplified model and the neglect of higher harmonics. The periodicity of the signal must be accurately represented by this approximation, however the amplitude dependence surely is not. The periodicity in steady flux has been examined to a fraction of a percent, but the periodicity in alternating flux has been confirmed to only a few percent. To this accuracy the period is $h / 2 e$. The absolute amplitude of the signal depends on feedback effects arising from the coupling of classical and quantum circuits, and has also been confirmed experimentally. As it stands these superconducting elements form a reliable basis for the development of a unique kind of instrumentation.

If the superconducting circuit contains two Dayem type bridges it becomes meaningful to consider current through the device, as well as the circulating current. This structure (see fig. 5) has many characteristics similar to the dc superconducting interferometer [12]. When the device is driven from a direct current source, above some critical current, voltage is produced roughly proportional to the excess current above critical :

$$
V=\alpha_{1}\left(I_{1}-I_{\mathrm{c} 1}(1)\right)=\alpha_{2}\left(I_{2}-I_{\mathrm{c} 1}(2)\right) .
$$

In addition, the sum of $I_{1}$ and $I_{2}$ is determined by the current source, $I_{1}+I_{2}=I$ and the average flux in the device is quantized :

$$
L\left(I_{1}-I_{2}\right)+\varphi_{\text {ext }}=N \varphi_{0}
$$

where $L$ is the inductance and $\varphi_{\text {ext }}$ is flux from some external source. Making the indicated substitutions the voltage becomes :

$$
V=\alpha\left\{\left(I / 2-I_{\mathrm{c}}\right)+\frac{\left(N \varphi_{0}-\varphi_{\mathrm{ext}}\right)}{2 L}\right\} .
$$

The quantum number $N$ will always adjust to keep $\left|N \varphi_{0}-\varphi_{\text {ext }}\right|$ as small as possible, leading to a voltage which is periodic influx, with period $\varphi_{0}$. The apparent discontinuous behavior at $\varphi_{\text {ext }}=(N+1 / 2) \varphi_{0}$ will 


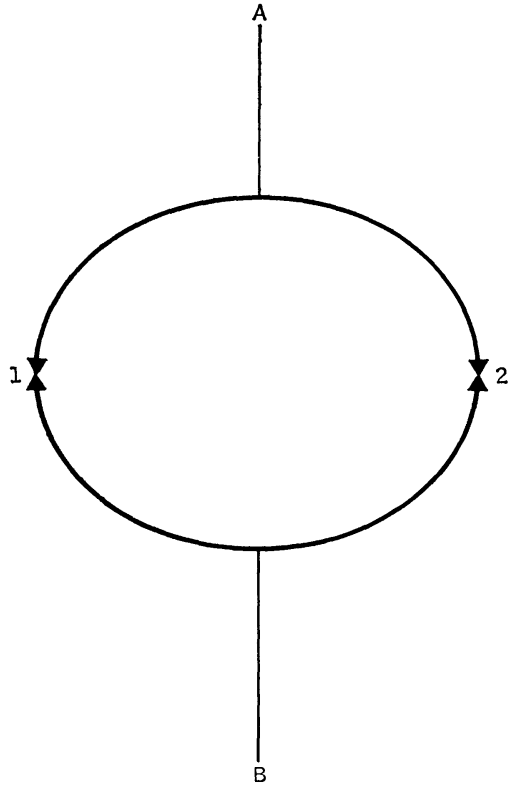

FIG. 5. - Schematic of a two bridge circuit where Dayem bridges at 1 and 2 are connected by superconducting links. This device is driven by a current source from $\mathrm{A}$ to $\mathrm{B}$.

always be thermally smeared [8] in practice, leading to a dc voltage whose amplitude is roughly sinusoidal in dc magnetic flux. Most of the "point contact" devices [13] probably also operate on principles similar to those discussed here.

Noise. - In this mode of operation the superconducting ring carries only supercurrent, except for the time interval of the transition, $\tau_{1}$. However, during this time, voltage appears and normal current with its attendant resistance must be developed. This appearance of resistance leads to an effective noise in the operation of the device.

During the transition we assume the superconducting circuit contains resistance $R(t)$. At constant resistance for this circuit, composed of an inductor and a resistor, the current noise is :

$$
I_{\mathrm{n}}^{2}(\omega)=\frac{2}{\pi} \frac{R k T \mathrm{~d} \omega}{R^{2}+\omega^{2} L^{2}} .
$$

This noise is spread over a frequency band-width roughly from zero to $R / L$ and has a total magnitude of :

$$
I_{\mathrm{n}}^{2}=\frac{2}{\pi} \int_{0}^{\infty} \frac{R k T \mathrm{~d} \omega}{R^{2}+\omega^{2} L^{2}}=\frac{k T}{L} .
$$

The total flux noise $\left(\varphi_{n}\right)^{2}$ from this current in a single turn is $\varphi_{\mathrm{n}}^{2}=L^{2} I^{2}=L k T$ and is independent of $R$. Thus, as the resistance drops and the ring tends toward complete superconductivity after the transition, this entire noise is confined to a decreasing bandwidth $(R / L)$, until as $R \rightarrow 0, L k T$ represents the uncertainty in the flux "trapped" by the ring at $R=0$. And $L k T$ also represents the spread expected in the magnitude of trapped flux in the ring if the trapping process is repeated many times.

Actually, since the flux trapped in a superconducting ring (inductance $L_{\mathrm{s}}$ ) is quantized, the trapped flux itself will always be quantized. This trapped flux is usually as close to the ambient value as can be achieved within the quantum rules. Adjacent values differing by $N \varphi_{0}$ are possible but are unlikely because of the extra energy involved. The result [8] is that the trapped flux is a periodic function of the ambient flux with period $\varphi_{0}$.

However in the presence of this noise the ambient value itself is uncertain by $\left(L_{\mathrm{s}} k T\right)^{\mathbf{1 / 2}}$. Thus the average trapped flux must be determined by averaging the ideal periodic behavior over a flux width of $L k T$. Clearly when $L k T \geqslant \varphi_{0}^{2}$ the periodicity disappears and quantum effects vanish.

The importance of this to the operation of these superconducting devices is to put a limit on the inductance of any device expected to show quantum behavior at $L_{\mathrm{s}} \lesssim \frac{\varphi_{0}^{2}}{k T}$ or $L_{\mathrm{s}} \lesssim 10^{-7} \mathrm{~h}$ at $4^{\circ} \mathrm{K}$. For a cylindrical ring, area $A$ and length $l$, inductance is given by $L=\frac{\mu A}{l}$. Thus for $l \simeq 10^{-3} \mathrm{~m}, A \leqslant 10^{-4} \mathrm{~m}^{2}$, or a maximum diameter of one $\mathrm{cm}$.

As magnetometers, the important parameter is equivalent field noise, $\delta B^{2}=\varphi_{n}^{2} / A^{2}$. Thus maximum sensitivity will be achieved at the largest size for which operation is possible. At this size the noise, $\varphi_{n}^{2}$, will be $\varphi_{0}^{2}$ so that in the above circuit $\delta B_{\min } \simeq 10^{-7} \mathrm{~g}$ spread over the current band-width. If this signal is examined with a time constant $\tau^{\prime}$ there will be an effective decrease in noise by a factor $\left(\tau^{\prime} \Omega / Q\right)^{1 / 2}$ where $\Omega$ is the resonance frequency. For most of our circuits $\Omega / Q \simeq 10^{5}$, thus a one second time constant implies a field noise of about $\delta B \simeq 3 \times 10^{-10} \mathrm{~g}$. Actually this can be improved somewhat by proper design of the circuit to increase its area while holding the inductance down. Our best sensitivity so far, with a one second time constant, is $7 \times 10^{-11} \mathrm{~g}$.

Another source of flux noise at the superconducting circuit is the input circuit itself. The equivalent input circuit for our amplifier is :

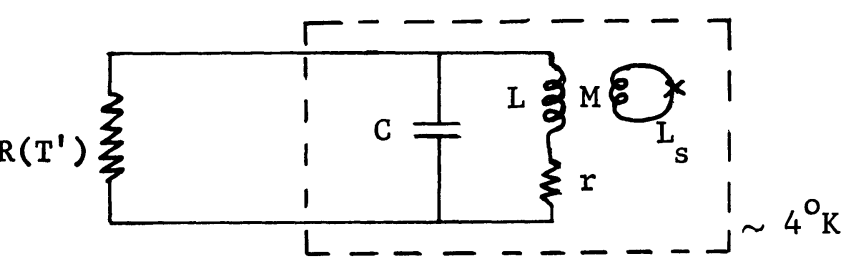

Current noise in this circuit is centered at the resonance frequency $\Omega=(L C)^{-1 / 2}$ and is limited by the $Q$ of the circuit to a frequency width roughly $\Omega Q^{-1}$. Most of the noise is generated in the room temperature resistor $R$ and is coupled to the superconducting circuit through mutual inductance $M$. The superconducting circuit will respond to all of the noise in the bandwidth of the input circuit or $\varphi_{\mathrm{n}}^{2}=M^{2} \int_{0}^{\infty} I_{\mathrm{n}}^{2} \mathrm{~d} \omega$. These noise signals are amplified and summed in a detector to give an equivalent input flux noise at the superconducting circuit of :

$$
\varphi_{\mathrm{n}}^{2}=\left(L_{\mathrm{s}} k T^{\prime}\right)\left(\frac{M^{2}}{L_{\mathrm{s}} L}\right)\left(Q^{2} \frac{r}{R}\right) .
$$

Here $T^{\prime}$ is the temperature of the resistance $R$. For most of our circuits the coupling $\left(\frac{M^{2}}{L_{\mathrm{s}} L}\right)$ is a few percent and $\left(Q^{2} \frac{r}{R}\right)$ is about one. Thus this flux 
noise in a circuit $1 \mathrm{~mm}$ in diameter $\left(L_{\mathrm{s}} \sim 10^{-9}\right)$ is about $1 / 10$ quantum.

It turns out that as long as this noise is relatively small $\left(<\varphi_{0}\right)$, the response of the superconductor to the noise depends strongly on the drive amplitude. Since the signal is quasi harmonic in $\varphi_{\mathrm{rf}}$ there are many signal maxima as a function of $\varphi_{\mathrm{rf}}$ (see fig. $4 \mathrm{~B}$ ). And, when the superconductor is biased at such a maximum, to first order the signal is insensitive to fluctuations in $\varphi_{\mathrm{rf}}$. Thus, biased to a point of maximum signal, the effect of noise is minimized. Conversely, by the same reasoning, at minimum signal the noise is greatest.

Figure 6 shows these effects. The variation of the superconducting emf with $\varphi_{\mathrm{dc}}$ is shown with $\varphi_{\mathrm{rf}}$ as a parameter. Note that at $2 \varphi_{\mathrm{rf}} \sim(2 n-1) \hbar / 2 e$ the

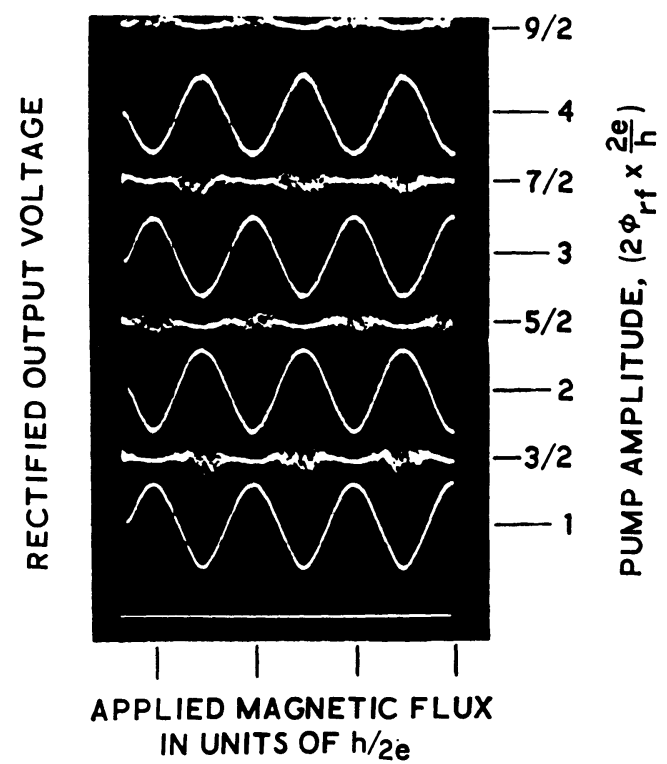

FIG. 6. - Amplitude of the superconducting emf as a function of steady magnetic field, $\varphi_{d c}$, with pump amplitude as a parameter.

signal is positive maximum, at $2 \varphi_{\mathrm{rf}} \sim 2 n \hbar / 2 e$ the signal is negative maximum, and in both cases the noise is small. While at $2 \varphi_{\mathrm{rf}} \sim \frac{2 n+1}{2} \hbar / 2 e$ the signal is zero and the noise is greatest. Note also the periodicity of both the signal and noise with $\varphi_{\mathrm{dc}}$. Actually it has been possible by proper choice of coupling and feedback to quiet the noise of the tank circuit. At the proper bias and coupling it is possible to achieve an induced signal from the tank circuit with considerably less noise than that generated by the tank itself.

Instrumentation - In our laboratory we have utilized these superconducting devices to produce highly sensitive ac and dc magnetometers, voltmeters and radiation detectors. I will concentrate here on the dc magnetometer applications. For this purpose the superconducting circuit is biased to some appropriate level such that the response to a change in dc field is as shown in figure $4 \mathrm{~A}$. Two types of magnetometer have been developed, an "analogue" and a "digital" instrument.

The "analogue", instrument [14] employs a feedback circuit which controls current to a solenoid surroun- ding (or through) the superconducting device. The magnetically controlled signal from the device is held constant (usually zero) by sensing any impending change in the signal and compensating for it by an appropriate current in the feedback solenoid. A determination of the field change is then made by measuring the control current for the feedback loop. The sensitivity of this instrument is determined by the field periodicity of the superconducting element and noise in the feedback loop. Devices with periodicity as low as $10^{-7}$ gauss have been used in feedback circuits able to control $\sim 10^{-3}$ of a period. The lowest noise device so far has produced an instrument whose sensitivity is about $7 \times 10^{-11}$ gauss with a one second time constant. The practical bandwidth for these feedback circuits is about 100 cycle $^{-1}$. This simple technique gives us a relatively sensitive magnetometer whose ultimate limit has not yet been achieved; the present limit is apparently set by noise in the feedback circuit.

One drawback of this previous technique is that the intrinsic periodicity of the signal (its quantum properties) are not utilized. And also that large field changes may go unobserved if they cause the instrument to "break lock" by exceeding the capabilities of the feedback loop and skip many periods. As described, the instrument cannot tell one signal zero from another. To overcome these difficulties a second type of instrument has been built to count flux periods [15]. By differentiating the signal (fig. $4 \mathrm{~A}$ ) twice and using these derivative signals to gate a counting circuit, both the sense and number of flux periods can be determined. This technique has been refined [16] so that now we have an instrument which will count in units of $1 / 4 \varphi_{0}$ at a rate of $10^{4}$ per s. Both of these magnetometers have been made into absolute instruments by the simple expedient of inverting the superconducting element [17] - the difference in readings being twice the absolute field.

These magnetometers can be converted to dc voltmeters by converting voltage to a known flux. This can be done by impressing the dc voltage on a $R-L$ series

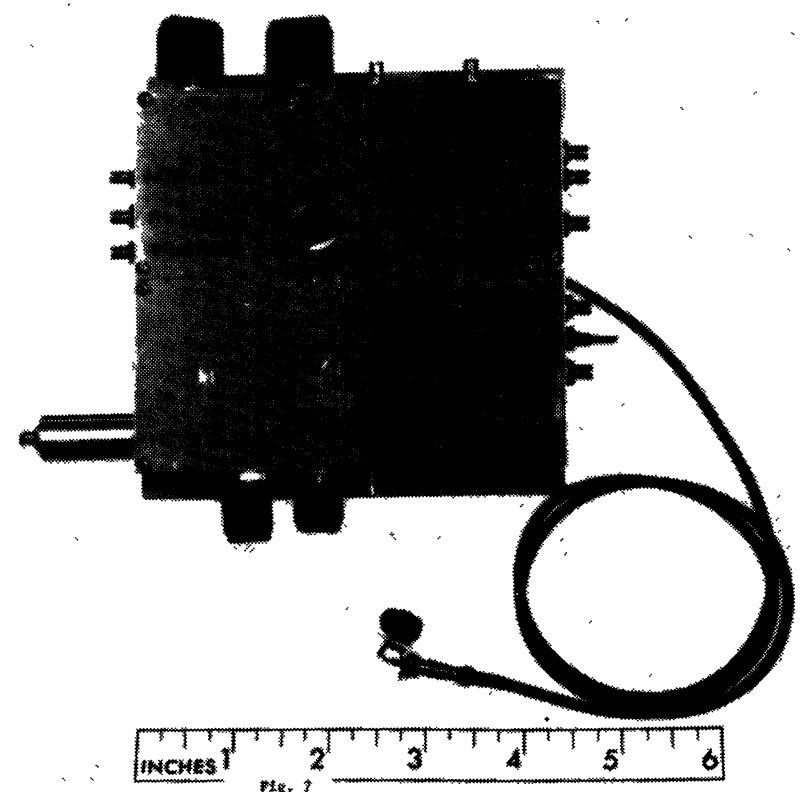

FIG. 7. - Complete instrument package. Superconducting circuit is contained within the tank coil at the end of the coaxial cable. 
circuit containing $N$ turns. If $R$ is much larger than the impedence of the voltage source, the current $I$ though this circuit is $V / R$. This current in turn generates a flux $\varphi=L I N^{-1}=\frac{L}{R} \frac{V}{N}$. The present magnetometer noise limit is about $10^{-3} \varphi_{0}$ giving an apparent voltage sensitivity of $V \sim N \frac{R}{L} 10^{-18} \mathrm{~V}$. However this circuit will develop a Johnson noise of its own where $\left(\varphi_{n}^{2}\right)=L k T N^{-2}$. This circuit noise will usually limit the flux sensitivity so that :

$$
\begin{gathered}
\frac{V^{2}}{N^{2}}\left(\frac{L}{R}\right)^{2} \sim L k T N^{-2} \\
V_{\mathrm{n}} \sim \frac{R}{L}(L k T)^{1 / 2} .
\end{gathered}
$$

Thus a micro-henry and a micro-ohm at $4.2 \mathrm{oK}$ will give a sensitivity of about $10^{-14} \mathrm{~V}$ and a time constant of one second.

Conclusion. - By the techniques which I have discussed in this paper we have made operating instru- ments which we are using in further laboratory experiments. The present sensitivity and response time of these instruments make them appropriate research tools for a diversity of experiments. Our use has ranged from determination of magnetic susceptibility [18] and measurements of fundamental constants [19] to a search for magnetic monopoles [20]. There seems no doubt that devices of this type can make further useful contributions to many other technical and scientific problems.

Our present "state of the art" in this cryogenic instrumentation is represented in figure 7. This package contains all the electronics required to drive and detect the superconducting thin film circuit I have discussed. Of course here the tank circuit and superconducting film at the end of the coaxial cable is the only element actually cooled to low temperatures. Despite repeated cooling to helium temperature these superconducting circuits nevertheless form reliable structures - some of our circuits have been successfully cooled hundreds of times over what is now a three year period.

\section{REFERENCES}

[1] KIm (Y. B.), HeMPSTEAD (C. F.) and Strnad (A. P.), Phys. Rev., 1963, 131, 2486.

Hempstead (C. F.) and KIm (Y. B.), Phys. Rev. Letters, 1964, 12, 145.

[2] Giaever (I.), Phys. Rev. Letters, 1965, 15, 825.

[3] Josephson (B. D.), Phys. Letters, 1962, 1, 251.

[4] Anderson (P. W.) and Dayem (A. H.), Phys. Rev. Letters, 1964, 13, 195.

[5] Clarke (J.), Proc. Roy. Soc. (to be published). FrIEBERTSHAUSER (P. E.), Notarys (H. A.) and Mercereau (J. E.), Bull. Am. Phys. Soc., Ser. II, 1968, 13, 1670.

[6] Bardeen (J.), Rev. Mod. Phys., 1962, 34, 667. Bhalnager (A. K.) and Stern (E. A.), Phys. Rev. Letters, 1968, 21, 1061.

[7] Fulde (P.) and Ferreil (R. A.), Phys. Rev., 1963, 131, 2457.

[8] Mercereau (J. E.), Proceedings of a Conference on Fluctuations in Superconductors, Asilomar, California, 1968.

[9] Mercereau (J. E.) and Crane (L. T.), Phys. Rev. Letters, 1962, 9, 381.
[10] Stephen (M. J.) and BARdeEN (J.), Phys. Rev. Letters, 1965, 14, 112.

[11] Mercereau (J. E.), Proceedings of Symposium on Physics of Superconducting Devices, Univ. of Virginia, Charlottesville, 1967.

[12] Jaklevic (R. C.), Lambe (J.), Silver (A. H.) and Mercereau (J. E.), Phys. Rev. Letters, 1964, 12, 159 .

[13] Zimmerman (J. E.) and Silver (A. H.), Phys. Rev., 1966, 141, 367 ; Phys. Rev., 1967, 157, 317.

[14] Forgacs (R. L.) and WarNick (A.), I.E.E.E. Transactions, 1966, IM-15, 113.

[15] Forgacs (R. L.) and WarnICK (A.), R.S.I., 1967, 38, 214.

[16] KELM (E.), Private communication.

[17] Simmonds (M.), Private communication.

[18] Vant-Hul, (L. L.) and Rossman (G.), Private communication.

[19] Simmonds (M.), PARker (W. K.) and Nisenoff (M.), Bull. Am. Phys. Soc., Series II, 1968, 13, 1668.

[20] Vant-Huli (L. L.), Phys. Rev., 1968, 173, 1412. 